\title{
Prescribing patterns of low doses of antipsychotic medications in older Asian patients with schizophrenia, 2001-2009
}

\author{
Yu-Tao Xiang, ${ }^{1,2}$ Faith Dickerson, ${ }^{3}$ Julie Kreyenbuhl, ${ }^{4,5}$ Gabor S. Ungvari, ${ }^{6}$ \\ Chuan-Yue Wang, ${ }^{2}$ Tian-Mei Si, ${ }^{7}$ Edwin H. M. Lee, ${ }^{1}$ Yan-Ling He, ${ }^{8}$ Helen F. K. Chiu, \\ Kelly Y. C. Lai, ${ }^{1}$ Naotaka Shinfuku, ${ }^{9}$ Shu-Yu Yang, ${ }^{10}$ Mian-Yoon Chong, ${ }^{11}$ Ee-Heok Kua, ${ }^{12}$ \\ Senta Fujii, ${ }^{13}$ Kang Sim, ${ }^{14}$ Michael K. H. Yong, ${ }^{14}$ Jitendra K. Trivedi, ${ }^{15}$ Eun-Kee Chung, ${ }^{16}$ \\ Pichet Udomratn, ${ }^{17}$ Kok-Yoon Chee, ${ }^{18}$ Norman Sartorius ${ }^{19}$ and Chay-Hoon Tan ${ }^{12}$ \\ ${ }^{1}$ Department of Psychiatry, Chinese University of Hong Kong, Hong Kong SAR, China \\ ${ }^{2}$ Beijing Anding Hospital, Capital Medical University, Beijing, China \\ ${ }^{3}$ The Stanley Research Program at Sheppard Pratt, Baltimore, Maryland, USA \\ ${ }^{4}$ Division of Services Research, Department of Psychiatry, University of Maryland School of Medicine, Baltimore, Maryland, USA \\ ${ }^{5}$ Veterans Administration Capitol Healthcare Network (VISN 5) Mental Illness Research, Education, and Clinical Center (MIRECC), Baltimore, \\ Maryland, USA \\ ${ }^{6}$ The University of Notre Dame Australia / Marian Centre, Perth, Australia \\ ${ }^{7}$ Key Laboratory of Mental Health, Ministry of Mental Health \& Peking University Institute of Mental Health, Beijing, China \\ ${ }^{8}$ Shanghai Mental Health Center, Shanghai, China \\ ${ }^{9}$ School of Human Sciences, Seinan Gakuin University Fukuoka, Japan \\ ${ }^{10}$ Taipei City Hospital, Taipei, Taiwan \\ ${ }^{11}$ Kaohsiung Chang Gung Memorial Hospital and School of Medicine, Chang Gung University, Taiwan \\ ${ }^{12}$ National University of Singapore, Singapore \\ ${ }^{13}$ Hyogo Institute for Traumatic Stress (HITS), Kobe, Japan \\ ${ }^{14}$ Institute of Mental Health, Buangkok View, Singapore \\ ${ }^{15}$ Department of Psychiatry, C.S.M. Medical University, Lucknow, Uttar Pradesh, India \\ ${ }^{16}$ National Seoul Hospital, Seoul, Korea \\ ${ }^{17}$ Department of Psychiatry, Faculty of Medicine, Prince of Songkla University, Songkhla, Thailand \\ ${ }^{18}$ Department of Psychiatry and Mental Health, Tunku Abdul Rahman Institute of Neuroscience, Kuala Lumpur Hospital, Malaysia \\ ${ }^{19}$ Association for the Improvement of Mental Health Programs, Geneva, Switzerland
}

ABSTRACT

Background: This study examined the use of low doses of antipsychotic medications (300 mg/day CPZeq or less) in older Asian patients with schizophrenia and its demographic and clinical correlates.

Methods: Information on hospitalized patients with schizophrenia, aged 55 years or older, was extracted from the database of the Research on Asian Psychotropic Prescription Patterns (REAP) study (2001-2009). Data on 1,452 patients in eight Asian countries and territories including China, Hong Kong, Japan, Korea, Singapore, Taiwan, India, and Malaysia were analyzed. Sociodemographic and clinical characteristics and antipsychotic prescriptions were recorded using a standardized protocol and data collection procedure.

Results: The prescription frequency for low doses of antipsychotic medications was $40.9 \%$ in the pooled sample. Multiple logistic regression analysis of the whole sample showed that patients on low doses of antipsychotic medications were more likely to be female, have an older age, a shorter length of illness, and less positive symptoms. Of patients in the six countries and territories that participated in all the surveys between 2001 and 2009, those in Japan were less likely to receive low doses of antipsychotics.

Conclusion: Low doses of antipsychotic medications were only applied in less than half of older Asian patients with schizophrenia.

Key words: schizophrenia, prescription patterns, low doses, older patients, Asia

\footnotetext{
Correspondence should be addressed to: Dr. Yu-Tao Xiang, Department of Psychiatry, Chinese University of Hong Kong, Ground Floor, Multicentre, Tai Po Hospital, Tai Po, N.T., Hong Kong. Phone: +852-2607-6041; Fax: +8522647-5321. Email: xyutly@gmail.com.; or Dr. Tian-Mei Si, Peking University Institute of Mental Health, Beijing, China. Email: si.tian-mei@163.com. Received 28 Sep 2011; revision requested 22 Nov 2011; revised version received 3 Dec 2011; accepted 13 Dec 2011. First published online 3 February 2012 .
}

\section{Introduction}

In the past decades, following the general improvement of mental healthcare and introduction of effective antipsychotics into clinical practice, many schizophrenia patients live into older adulthood (Kohen et al., 2010). Given the higher 
likelihood of drug-induced side effects and poor general health in older compared to younger patients (Meyers and Jeste, 2010), rational use of antipsychotics in this population has been gaining increasing attention.

Neuroimaging studies demonstrate that dopamine-2 occupancy, one of the principal targets of antipsychotics, decreases $5 \%-10 \%$ per decade with age, incurring the likelihood of greater susceptibility for antipsychotic-induced adverse effects (Uchida et al., 2009a). In addition, age-related pharmacokinetic changes affect drug absorption and excretion, which may also exacerbate adverse effects (Masand, 2000). Therefore, expert consensus guidelines suggest the use of lower doses of antipsychotics in older patients (Alexopoulos et al., 2004); for example, whereas the recommended therapeutic dose of risperidone is $4-8 \mathrm{mg}$ for younger adult schizophrenia patients, expert consensus guidelines suggest a range of only $1.25-3.5 \mathrm{mg}$ for older patients (Alexopoulos et al., 2004). To date, however, no large-scale, pharmaco-epidemiological surveys on patterns of antipsychotic dosing in older patients with schizophrenia have been reported in Asia.

In order to rationalize the use of psychotropic medications in Asian countries, a large-scale longitudinal, observational pharmaco-epidemiological study titled Research on Asian Psychotropic Prescription Pattern (REAP) was initiated in 1999 in six Asian countries and territories including China, Hong Kong, Japan, Korea, Singapore, and Taiwan, and investigated prescription trends for psychotropic medications in hospitalized patients with schizophrenia in Asia.

This study is a secondary analysis of the data of the REAP project which aimed to (1) examine the prescribing pattern of low doses of antipsychotics (defined as $300 \mathrm{mg} /$ day chlorpromazine equivalent (CPZeq) or less) (Sim et al., 2009) in older Asian patients with schizophrenia during the period between 2001 and 2009, and (2) survey its demographic and clinical correlates. Due to the poorer general health status of older patients and heightened vulnerability to psychotropic-induced side effects (Uchida et al., 2009b; Meyers and Jeste, 2010), we hypothesized that the majority of older patients with schizophrenia would be prescribed low doses of antipsychotics in the REAP project.

\section{Methods}

\section{Settings, study design, and subjects}

The first survey of the REAP project was carried out in July 2001 followed by investigations in July 2004 and October 2008 to March 2009 with
Table 1. Commonly used antipsychotic medications and their chlorpromazine equivalent (CPZeq) in milligrams per day (APA, 1997; Kane et al., 1998; Woods, 2003)

\begin{tabular}{|c|c|}
\hline ANTIPSYCHOTICS & $\begin{array}{l}\text { CPZeq } \\
\text { (mg/day) }\end{array}$ \\
\hline \multicolumn{2}{|c|}{ First-generation antipsychotic medications } \\
\hline Chlorpromazine & 100 \\
\hline Haloperidol & 2 \\
\hline Levomepromazine & 100 \\
\hline Sulpiride & 200 \\
\hline Trifluoperazine & 5 \\
\hline \multicolumn{2}{|c|}{ Second-generation antipsychotic medications } \\
\hline Risperidone & 2 \\
\hline Clozapine & 50 \\
\hline Olanzapine & 5 \\
\hline Quetiapine & 75 \\
\hline Zotepine & 66 \\
\hline
\end{tabular}

the same study design and standardized protocol. Centers in India, Malaysia, and Thailand joined the surveys in 2009. Details of the REAP project have been described elsewhere (Chong et al., 2004; Sim et al., 2004; Shinfuku and Tan, 2008) and are summarized as follows. Data of patients were analyzed in this study if they satisfied the following criteria: (1) ICD-10 or DSM-IV schizophrenia, (2) aged 55 years or older, and (3) willingness to provide written or oral consent in case the consent was not waived according to the requirements of the Clinical Research Ethics Committee in the respective study sites. Patients with significant medical conditions were excluded. Doses of antipsychotic drugs were converted into CPZeq milligrams (APA, 1997; Kane et al., 1998; Woods, 2003) (see Table 1). Following an earlier study (Sim et al., 2009), a low dose of antipsychotics was defined as $300 \mathrm{mg} /$ day CPZeq or less.

Eligible patients were recruited consecutively and their sociodemographic and clinical characteristics including age, sex, length of illness, type, and doses of antipsychotic medications, the presence or absence of significant psychotic symptoms in the past month, extrapyramidal side effects (EPS) and tardive dyskinesia (TD) were collected by a review of medical records in 2001, and by either a review of medical records only or a review of medical records supplemented by a patient interview in 2004 and 2009 using a questionnaire designed for the study. The data were collected by the attending psychiatrists or by members of the research team with the agreement of the patient's treating psychiatrist.

The study was approved by the Clinical Research Ethics Committees of the respective centers. Given the anonymous nature of this observational study 
and minimal risk to patients, the patients' informed consent was exempted in some participating study sites according to the requirements of the local Clinical Research Ethics Committee (Shinfuku and Tan, 2008). The requirements of the Clinical Research Ethics Committee usually vary on a local basis.

\section{Statistical analysis}

The data were analyzed using SPSS 13.0 for Windows (SPSS, Inc., Chicago, IL). Comparisons of low doses of antipsychotics across the three surveys were made with $\chi^{2}$ tests. Multiple logistic regression analysis was used to determine the demographic and clinical variables influencing the receipt of low doses of antipsychotics in the pooled sample. Cross-sectional low dose of antipsychotics was the dependent variable, while independent variables included study site and survey time point, age, gender, symptom severity, length of illness, the presence of EPS and TD, and use of antipsychotics. The level of significance was set at 0.05 (two-tailed).

\section{Results}

Thirty-one psychiatric institutions were involved in 2001, 25 in 2004, and 50 in 2009. A total of 1,452 patients met the study inclusion criteria; 490 in 2001, 446 in 2004, and 516 in 2009. A total of 594 patients of the 1,452 received low doses of antipsychotics $(40.9 \%)$ in the three REAP surveys; 203 in 2001 (41.4\%), 192 in $2004(43.0 \%)$, and 199 in $2009(38.6 \%)$. There was no significant difference across the three surveys in the use of low doses of antipsychotics $\left(\chi^{2}=2.1, d f=2, \mathrm{p}=0.4\right)$. Of the patients on first-generation antipsychotics (FGAs) only $(n=561), 229(40.8 \%)$ received low doses of antipsychotics; in patients on secondgeneration antipsychotics (SGAs) only $(n=514)$ $56.2 \%(n=289)$ received them. In contrast, only $55(15.6 \%)$ of those prescribed combinations of FGAs and SGAs $(n=353)$ received doses less than 300 CPZeq. Table 2 shows the sociodemographic and clinical characteristics of the whole sample and separately for patients by study site. Figure 1 presents the use of low doses of antipsychotics in participating countries and regions over the study period.

Table 3 displays the factors that were independently associated with low doses of antipsychotics. Patients on low doses of antipsychotics were more likely to be female, have an older age, a shorter length of illness, and less positive symptoms. Of patients in the six countries and territories that participated in the three surveys, those in Japan were less likely to receive low doses of antipsychotics.

\section{Discussion}

Treatment recommendation in the USA suggests that maintenance doses of antipsychotics below $300 \mathrm{mg} /$ day CPZeq usually increase the risk of relapse (Lehman et al., 2004). Based on the currently prevailing view, however, Asian patients require lower doses of antipsychotics and they are more sensitive to EPS than their Western counterparts (Chiu et al., 1991; Frackiewicz et al., 1997). For example, Chow et al. (1999) reported that schizophrenia patients could be maintained on a median daily dose of $300 \mathrm{mg}$ CPZeq of antipsychotic medications in Hong Kong. In another study, Okuma et al. (1981) found that a median daily dose of $270 \mathrm{mg}$ CPZeq was the usual dose in maintenance treatment of Japanese patients with schizophrenia. Considering that antipsychotic level may be also affected by age-related changes in renal excretion and hepatic metabolism in the elderly, even lower doses of antipsychotics should likely be considered in Asian older patients with schizophrenia.

To the best of our knowledge, there has been no previous international study investigating the use of low doses of antipsychotics in older patients with schizophrenia. The assumption that the majority of older patients with schizophrenia would receive low doses of antipsychotics in the current sample was not supported. The actual mean daily dose of antipsychotics was $556 \mathrm{mg}$ CPZeq, and only $40.9 \%$ of the patients were given low doses of antipsychotics. Nearly half $(50.1 \% ; 234 / 467)$ patients aged 65 or older the standard age definition for psychogeriatric patients in the international literature - received low doses of antipsychotics, a figure that also fails to support our hypothesis. The percentage of low antipsychotic doses was particularly small in Japan and India, although only a very small sample from the latter country was involved. The reasons for these differences between countries and for the limited use of low antipsychotic doses in older Asian patients need to be explored in future studies.

In this study, independent correlates of low doses of antipsychotics included being female, being older, having a shorter length of illness, and having less positive symptoms. There are significant sex differences in pharmacodynamics, pharmacokinetics, and subjective tolerability to antipsychotic drugs (Seeman, 2010). Relatively younger patients often present with more aggressive and impulsive behaviors (Alexopoulos et al., 2004). These two factors could possibly explain the association of male sex, and relatively younger age, with less use of low antipsychotic doses. Refractory symptoms are more often displayed 
Table 2. Sociodemographic and clinical characteristics of older Asian patients with schizophrenia in REAP surveys 2001-2009*

\begin{tabular}{|c|c|c|c|c|c|c|c|c|c|c|c|c|c|c|c|c|c|c|}
\hline & \multicolumn{2}{|c|}{$\begin{array}{l}\text { CHINA } \\
(n=215)\end{array}$} & \multicolumn{2}{|c|}{$\begin{array}{l}\text { HONG } \\
\text { KONG } \\
(n=43)\end{array}$} & \multicolumn{2}{|c|}{$\begin{array}{l}\text { INDIA } \\
(n=5)\end{array}$} & \multicolumn{2}{|c|}{$\begin{array}{l}\text { JAPAN } \\
(n=826)\end{array}$} & \multicolumn{2}{|c|}{$\begin{array}{l}\text { KOREA } \\
(n=128)\end{array}$} & \multicolumn{2}{|c|}{$\begin{array}{l}\text { SINGAPORE } \\
(n=84)\end{array}$} & \multicolumn{2}{|c|}{$\begin{array}{l}\text { TAIWAN } \\
(n=143)\end{array}$} & \multicolumn{2}{|c|}{$\begin{array}{l}\text { MALAYSIA } \\
(n=8)\end{array}$} & \multicolumn{2}{|c|}{$\begin{array}{l}\text { TOTAL } \\
(n=1452)\end{array}$} \\
\hline & MEAN & $\mathrm{SD}$ & MEAN & SD & MEAN & SD & MEAN & SD & MEAN & SD & MEAN & $\mathrm{SD}$ & MEAN & SD & MEAN & SD & MEAN & SD \\
\hline Age (yrs) & 59.5 & 5.3 & 62.7 & 6.4 & 57.0 & 2.1 & 64.2 & 6.8 & 59.8 & 4.5 & 60.6 & 4.8 & 60.1 & 5.4 & 58.8 & 3.5 & 62.4 & 6.5 \\
\hline \multirow[t]{2}{*}{ CPZeq (mg/d) } & 431 & 334 & 404 & 379 & 508 & 163 & 636 & 616 & 577 & 528 & 429 & 410 & 394 & 311 & 331 & 175 & 556 & 539 \\
\hline & $N$ & $\%$ & $N$ & $\%$ & $N$ & $\%$ & $N$ & $\%$ & $N$ & $\%$ & $N$ & $\%$ & $N$ & $\%$ & $N$ & $\%$ & $N$ & $\%$ \\
\hline Length of illness ( $<5$ years) & 19 & 8.8 & 3 & 7.0 & 1 & 20.0 & 16 & 1.9 & 5 & 3.9 & 6 & 7.1 & 3 & 2.1 & 1 & 12.5 & 54 & 3 \\
\hline Men & 114 & 53.0 & 23 & 53.5 & 3 & 60.0 & 436 & 52.8 & 54 & 42.2 & 45 & 53.6 & 81 & 56.6 & 4 & 50.0 & 760 & 52 \\
\hline Positive symptoms & 116 & 54.0 & 25 & 58.1 & 5 & 100 & 562 & 68.0 & 90 & 70.3 & 39 & 46.4 & 93 & 65.0 & 5 & 62.5 & 935 & 64. \\
\hline Negative symptoms & 151 & 70.2 & 28 & 65.1 & 4 & 80.0 & 597 & 72.3 & 64 & 50.0 & 34 & 40.5 & 86 & 60.1 & 1 & 12.5 & 965 & 66. \\
\hline EPS & 32 & 14.9 & 24 & 55.8 & 1 & 20.0 & 270 & 32.7 & 38 & 29.7 & 8 & 9.5 & 56 & 39.2 & 4 & 50.0 & 433 & 29.8 \\
\hline TD & 13 & 6.0 & 11 & 25.6 & 0 & 0 & 74 & 9.0 & 6 & 4.7 & 3 & 3.6 & 23 & 16.1 & 2 & 25.0 & 132 & 9 \\
\hline FGAs $^{1}$ & 82 & 38.1 & 19 & 44.2 & 2 & 40.0 & 588 & 71.2 & 89 & 69.5 & 72 & 85.7 & 56 & 39.2 & 6 & 75.0 & 914 & 62.9 \\
\hline SGAs $^{2}$ & 161 & 74.9 & 22 & 51.2 & 4 & 80.0 & 513 & 62.1 & 61 & 47.7 & 7 & 8.3 & 97 & 67.8 & 2 & 25.0 & 867 & 59.7 \\
\hline Low doses & 104 & 48.4 & 22 & 51.2 & 0 & 0 & 291 & 35.2 & 49 & 38.3 & 45 & 53.6 & 78 & 54.5 & 5 & 62.5 & 594 & 40.9 \\
\hline
\end{tabular}

* There were no older patients in Thailand

1 any use of FGAs; ${ }^{2}$ any use of SGAs

$\mathrm{CPZeq}=$ chlorpromazine equivalents; $\mathrm{EPS}=$ extrapyramidal symptoms; $\mathrm{TD}=$ tardive dyskinesia; FGA $=$ first-generation antipsychotic; $\mathrm{SGA}=$ second-generation antipsychotic. 


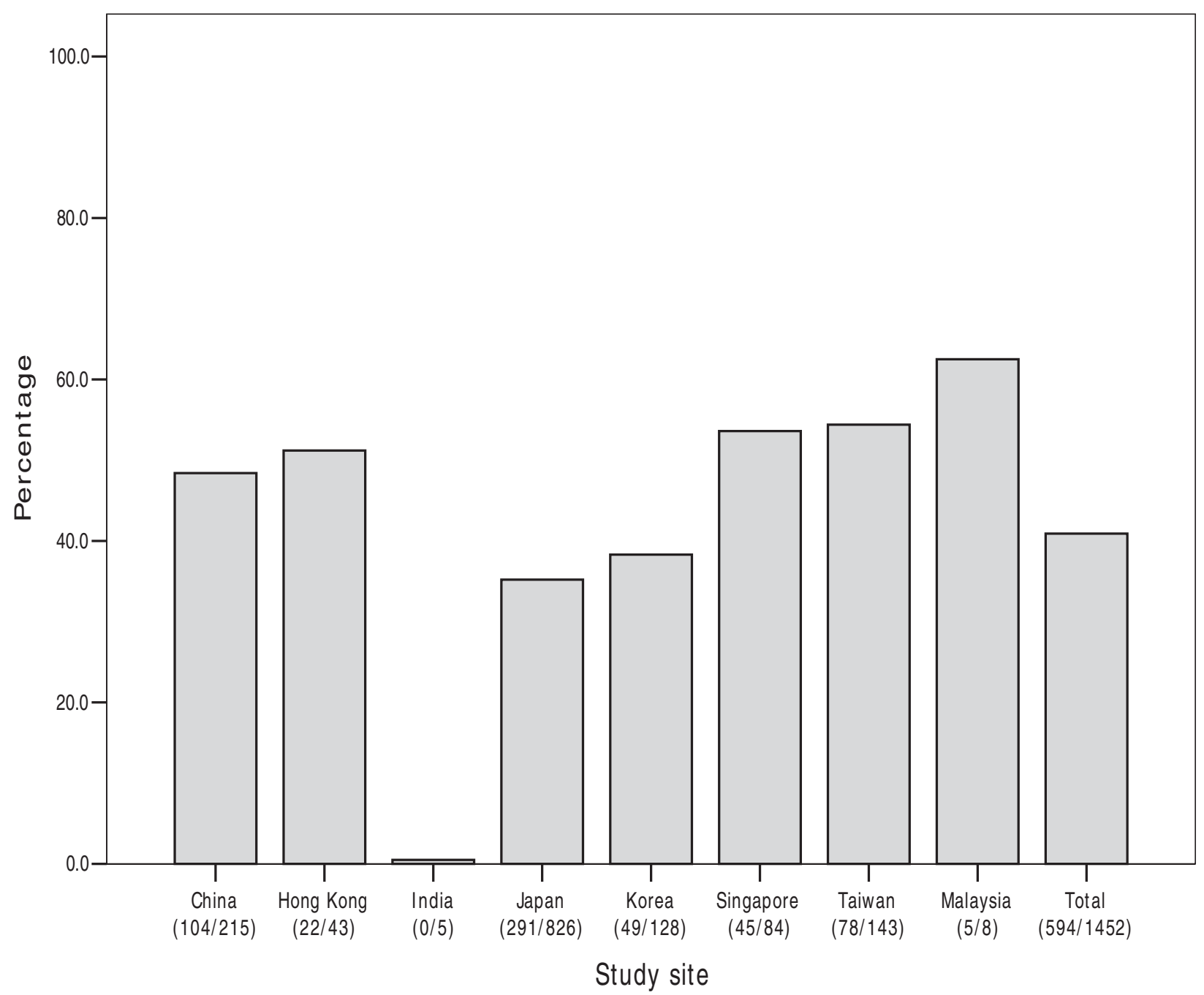

Figure 1. Percentage of older patients with schizophrenia receiving low doses of antipsychotic medications.

by hospitalized patients with a longer length of illness, which could possibly explain the relationship between shorter length of illness and low doses of antipsychotics. Patients with prominent positive symptoms were more likely to receive relatively higher doses of antipsychotics in this study, which might be explained by the false assumption that higher doses can better optimize the dopamine2 receptor occupancy ( $\mathrm{Ji}, 2005)$. In addition, we found that, not surprisingly, patients who were prescribed a combination of FGAs and SGAs were less likely to receive a low dose of antipsychotics relative to those prescribed monotherapy, which is in line with an earlier finding (Barbui et al., 2006).

There are a number of limitations to this study. First, the study targeted inpatients in eight selected Asian countries and territories in this huge continent with a wide variety of sociocultural, economic, and mental healthcare systems; therefore, the findings cannot be applied to all schizophrenia patients in Asia. Second, due to the cross-sectional design, the causal relationship between use of low antipsychotic doses and demographic and clinical variables cannot be explored. Third, a host of important variables likely to influence appropriate prescription, such as the length of antipsychotic treatment prior to the study and that of the schizophrenic illness, the effectiveness and the benefit/risk ratio of current prescriptions, separate examination of lateonset schizophrenia, local prescription guidelines, drug interactions, smoking, the type of psychiatric facilities, and mental healthcare policies were not evaluated. Fourth, length of the current admission was not taken into account in this study although it may have an impact on dosing; many clinicians titrate slowly in older patients ("start low, go slow"), which would influence cross-sectional evaluation of prescribing patterns. Fifth, the conversion of antipsychotic doses into CPZeq is not accurate, particularly for SGAs. Using the same conversion standard when comparing the two samples (lower dose vs. higher dose group) in the multivariate analysis could mitigate this limitation as it may 
Table 3. Sociodemographic and clinical correlates associated with low antipsychotic doses in the combined sample $(n=1,439)$. Multiple logistic regression analysis with higher doses of antipsychotic medications (more than $300 \mathrm{mg} /$ day CPZeq) as the reference group

\begin{tabular}{|c|c|c|c|}
\hline & P VALUE & OR & $95 \% \mathrm{CI}$ \\
\hline Age (year) & $<0.001$ & 1.1 & $1.06-1.1$ \\
\hline Male sex & 0.001 & 0.7 & $0.6-0.9$ \\
\hline Length of illness & 0.02 & 0.5 & $0.3-0.9$ \\
\hline Positive symptoms & 0.001 & 0.7 & $0.5-0.9$ \\
\hline Negative symptoms & 0.3 & 0.9 & $0.7-1.1$ \\
\hline EPS & 0.6 & 1.1 & $0.8-1.4$ \\
\hline $\mathrm{TD}$ & 0.2 & 1.3 & $0.9-2.0$ \\
\hline SGAs ${ }^{1}$ & 0.7 & 0.9 & $0.7-1.2$ \\
\hline \multicolumn{4}{|l|}{ Study sites } \\
\hline China & - & 1.0 & - \\
\hline Hong Kong & 0.6 & 0.8 & $0.4-1.7$ \\
\hline Japan & $<0.001$ & 0.4 & $0.3-0.6$ \\
\hline Korea & 0.06 & 0.6 & $0.4-1.02$ \\
\hline Singapore & 0.9 & 1.0 & $0.6-1.8$ \\
\hline Taiwan & 0.2 & 1.4 & $0.9-2.1$ \\
\hline \multicolumn{4}{|l|}{ Study time } \\
\hline 2001 survey & - & 1.0 & - \\
\hline 2004 survey & 0.4 & 1.1 & $0.8-1.5$ \\
\hline 2009 survey & 0.5 & 0.9 & $0.7-1.2$ \\
\hline
\end{tabular}

Centers in India, Malaysia, and Thailand joined the survey in 2009; therefore, they were not included in multiple logistic regression analysis. There was co-linearity between the use of FGA and SGA; therefore use of FGA was not included in multiple logistic regression analysis.

$\mathrm{OR}=$ odds ratio EPS $=$ extrapyramidal symptoms; $\mathrm{TD}=$ tardive dyskinesia; SGA = second-generation antipsychotic medication.

${ }^{1}$ any use of SGAs.

reveal relative trends in prescription practices (Ungvari et al., 2002). Finally, psychopathology and drug-induced side effects were not assessed using standardized rating instruments. The limitations of the present study are partly offset by its strengths including the large sample size and number of participating sites.

In conclusion, the findings of our study suggest that low doses of antipsychotics for Asian older patients with schizophrenia were prescribed only to a minority of patients overall and to an even smaller percentage of those prescribed more than one antipsychotic, a practice with limited evidence for its efficacy (Kreyenbuhl et al., 2010). Use of the lowest effective dose of antipsychotics in older individuals with schizophrenia should be encouraged in daily clinical practice in order to maximize the safety and tolerability of treatment in this at-risk population.

It should be noted that there are no gold-standard treatment guidelines for the use of antipsychotic drugs. Dose adjustment should be individualized and patients' clinical condition should be carefully considered. For older patients, the old clinical wisdom of "start low and go slow" principle is probably still the best advice.

\section{Conflict of interest}

None.

\section{Description of authors' roles}

Naotaka Shinfuku, Chay-Hoon Tan, Mian-Yoon Chong, Ee-Heok Kua, Gabor S. Ungvari and Norman Sartorius designed the study. Yu-Tao Xiang, Chuan-Yue Wang, Tian-Mei Si, and Gabor $\mathrm{S}$. Ungvari undertook all statistical analyses and helped with their interpretation. Yu-Tao Xiang and Gabor S. Ungvari wrote the first draft of the paper. Faith Dickerson, Julie Kreyenbuhl, ChuanYue Wang, Tian-Mei Si, Edwin HM Lee, Yan-Ling He, Gabor S. Ungvari, Helen F.K. Chiu, Kelly Y. C. Lai, Shu-Yu Yang, Mian-Yoon Chong, Naotaka Shinfuku, Chay Hoon Tan, Ee Heok Kua, Senta Fujii, Kang Sim, Michael K.H. Yong, Jitendra K. Trivedi, Eun-Kee Chung, Pichet Udomratn, KokYoon Chee, and Norman Sartorius contributed to the final writing of the paper. Naotaka Shinfuku, Mian-Yoon Chong and Shu-Yu Yang provided administrative, technical, or material support. All authors contributed to the final paper.

\section{Acknowledgments}

This study was supported in part by grants from the National Natural Science Foundation of China (81171270; 30800367; 30770776), the Beijing Nova Program of the Beijing Municipal Science and Technology Commission (2008B59), the Chinese University of Hong Kong (Direct Grant for Research; Project 2041454), the Institute of Mental Health Research Grant (CRC 249/2008) in Singapore, the Taiwan Bureau of National Health Insurance (DOH92-NH-1025), Chang Gung Memorial Hospital (CMRPG83043), and the Taipei City Government (97001-62-010) in Taiwan. The authors are grateful to the following clinicians involved in the data collection: Hong Deng and Wei Hao in China; Ajit Avasthi, Dipesh Bhagabati, Roy Abraham Kallivayalil, Shubhangi R. Parkar, and YC Janardhan Reddy in India; Tateno Masaru, Masamune Yayoi, Akiyama Tsuyoshi, Sato Soichirou, Nakagome Kazuyuki, Nakamura Jun, and Kuroki Toshihide in Japan; Tae-Yeon Hwang, Seok Hyeon Kim, Yo Wang Lee, and Jong- Il Lee in Korea; Tung-ping Su, Shih-ku Lin, Tzu-ting Chen, Chieh-hsin Chang, Hong-chieh Hsu, Chi-Fa Hung, and Chengchung Chen in Taiwan; Krisakorn Sukavatvibul, 
Jittima Kleawtanong, Tantawan Suradechasakul, Manote Lotrakul, and Usaree Srisutudsanavong in Thailand; and Norharlina Bahar in Malaysia. The authors also thank the clinicians who helped to organize the study at each study site.

\section{References}

Alexopoulos, G. S., Streim, J., Carpenter, D. and Docherty, J. P. (2004). Using antipsychotic agents in older patients. Fournal of Clinical Psychiatry, 65 (Suppl. 2), 5-99; discussion 100-102; quiz 103-104.

APA (1997). Practice Guideline for the Treatment of Patients with Schizophrenia. Washington, DC: American Psychiatric Press.

Barbui, C. et al. (2006). Persistence with polypharmacy and excessive dosing in patients with schizophrenia treated in four European countries. International Clinical Psychopharmacology, 21, 355-362.

Chiu, H. F., Shum, P. S. and Lam, C. W. (1991). Psychotropic drug prescribing to chronic schizophrenics in a Hong Kong hospital. International fournal of Social Psychiatry, 37, 187-191.

Chong, M. Y. et al. (2004). Antipsychotic drug prescription for schizophrenia in East Asia: rationale for change. Psychiatry and Clinical Neurosciences, 58, 61-67.

Chow, L. Y., Ungvari, G. S., Chiu, H. F. K. and Leung, T. (1999). Psychotropic drug prescription for chronic psychiatric pateints in a long-stay care facility. Hong Kong Fournal of Psychiatry, 9, 15-20.

Frackiewicz, E. J., Sramek, J. J., Herrera, J. M., Kurtz, N. M. and Cutler, N. R. (1997). Ethnicity and antipsychotic response. Annals of Pharmacotherapy, 31, 1360-1369.

Ji, Z. F. (2005). Evaluation and analysis of drug-use in hospitals of China. Evaluation of Clinical Usage of Partial Dopamine Receptor Agonist, 5, 89-91 [in Chinese].

Kane, J. M. et al. (1998). Guidelines for depot antipsychotic treatment in schizophrenia. European neuropsychopharmacology consensus conference in Siena, Italy. European Neuropsychopharmacology, 8, 55-66.

Kohen, I., Lester, P. E. and Lam, S. (2010). Antipsychotic treatments for the elderly: efficacy and safety of aripiprazole. Neuropsychiatric Disease and Treatment, 6, 47-58.
Kreyenbuhl, J., Buchanan, R. W., Dickerson, F. B. and Dixon, L. B. (2010). The Schizophrenia Patient Outcomes Research Team (PORT): updated treatment recommendations 2009. Schizophrenia Bulletin, 36, 94-103.

Lehman, A. F. et al. (2004). The Schizophrenia Patient Outcomes Research Team (PORT): updated treatment recommendations 2003. Schizophrenia Bulletin, 30, 193-217.

Masand, P. S. (2000). Side effects of antipsychotics in the elderly. Fournal of Clinical Psychiatry, 61 (Suppl. 8), 43-49.

Meyers, B. S. and Jeste, D. V. (2010). Geriatric psychopharmacology: evolution of a discipline. Fournal of Clinical Psychiatry, 71, 1416-1424.

Okuma, T. (1981). Differential sensitivity to the effects of psychotropic drugs: psychotics vs normals; Asian vs Western populations. Folia Psychiatrica et Neurologica faponica, 35, 79-87.

Seeman, M. V. (2010). Schizophrenia: women bear a disproportionate toll of antipsychotic side effects. Fournal of the American Psychiatric Nurses Association, 16, 21-29.

Shinfuku, N. and Tan, C. H. (2008). Pharmacotherapy for schizophrenic inpatients in East Asia - changes and challenges. International Review of Psychiatry, 20, 460-468.

Sim, K. et al. (2004). Antipsychotic polypharmacy in patients with schizophrenia: a multicentre comparative study in East Asia. British fournal of Clinical Pharmacology, $58,178-183$.

Sim, K. et al. (2009). Low doses of antipsychotic drugs for hospitalized schizophrenia patients in East Asia: 2004 vs. 2001. International fournal of Neuropsychopharmacology, 12, 117-123.

Uchida, H., Mamo, D. C., Mulsant, B. H., Pollock, B. G. and Kapur, S. (2009a). Increased antipsychotic sensitivity in elderly patients: evidence and mechanisms. Fournal of Clinical Psychiatry, 70, 397-405.

Uchida, H., Pollock, B. G., Bies, R. R. and Mamo, D. C. (2009b). Predicting age-specific dosing of antipsychotics. Clinical Pharmacology and Therapeutics, 86, 360-362.

Ungvari, G. S., Chung, Y. G., Chee, Y. K., Fung-Shing, N., Kwong, T. W. and Chiu, H. F. (2002). The pharmacological treatment of schizophrenia in Chinese patients: a comparison of prescription patterns between 1996 and 1999. British fournal of Clinical Pharmacology, 54, 437-444.

Woods, S. W. (2003). Chlorpromazine equivalent doses for the newer atypical antipsychotics. Fournal of Clinical Psychiatry, 64, 663-667. 